\title{
Desafíos y oportunidades en tiempos del COVID-19: contexto pedagógico desde la Universidad Nacional y la Universidad Estatal a Distancia
}

\author{
Challenges and Opportunities during COVID-19: Pedagogical Context \\ at Universidad Nacional and Universidad Estatal a Distancia
Desafios e oportunidades em tempos de COVID-19: contexto pedagógico da Universidad Nacional e da Universidad Estatal a Distancia

\author{
Priscilla Carranza-Marchena \\ Universidad Nacional \\ Heredia, Costa Rica \\ priscilla.carranza.marchena@una.ac.cr \\ (D) https://orcid.org/0000-0002-0742-7328 \\ Geraldine Zamora-Sánchez \\ Universidad Estatal a Distancia \\ San José, Costa Rica \\ gzamora@uned.ac.cr \\ (iD https://orcid.org/0000-0001-6471-9231
}

Recibido - Received - Recebido: 13 / 07 / 2020 Corregido - Revised - Revisado: 27 / 08 / 2020 Aceptado-Accepted - Aprovado: 02 / 09 / 2020

\author{
DOI: https://doi.org/10.22458/ie.v22iespecial.3154 \\ URL: https://revistas.uned.ac.cr/index.php/innovaciones/article/view/3154
}

\begin{abstract}
Resumen: Durante la pandemia generada por el virus responsable del COVID-19, el sistema educativo dio un giro rotundo de la presencialidad tradicional a la remota; esto por medio de la utilización de recursos tecnológicos y diversas plataformas, las cuales permitieron la continuidad del proceso educativo. Este suceso evidenció que los modelos pedagógicos que estaban siendo implementados no respondían a las necesidades educativas de progreso y de transformación digital generadas durante la crisis sanitaria. Por lo tanto, el presente ensayo tiene como objetivo primordial reflexionar acerca de los desafíos y oportunidades de mejora que el profesorado enfrentó a causa de la pandemia. El mismo se desarrolla bajo un enfoque interpretativo en el cual se discuten tres desafíos educativos: (1) el cambio contextual, es decir, la migración de las aulas a plataformas digitales y su impacto en la comunicación entre el personal docente y el estudiantado; (2) el aprendizaje acelerado en el ámbito tecnológico representó un segundo reto para el profesorado ante la carencia, en términos generales, de capacidades tecnológicas para la mediación pedagógica, esto previo a la pandemia y (3) la escogencia de herramientas tecnológicas efectivas que fueran de acceso libre y de fácil utilización para el fortalecimiento de un aprendizaje significativo. Este ensayo concluye con pensamientos reflexivos que reafirman el quehacer académico y los desafíos durante la pandemia, así como oportunidades de mejora en relación con la adaptación, el cambio, y la modernización en un ambiente turbulento y de incertidumbre.
\end{abstract}

Palabras clave: ambiente educacional, competencias del docente, tecnología, oportunidades educacionales, brecha digital, COVID-19

Summary: During the pandemic caused by the virus COVID-19, the educational system experienced a dramatic change from face-to-face to remote learning through the use of technological resources and various platforms to continue with the educational process. This event showed that the pedagogical models that were being implemented did not address the educational needs related to the progress and digital transformation throughout the health crisis. Therefore, this essay aims to reflect on the challenges and opportunities for improvement professors faced due to the pandemic. In fact, this interpretative essay discusses three educational challenges: (1) the contextual change, meaning, the migration from the classrooms to digital platforms and its impact in terms of the communication between instructors and students. (2) The accelerated learning process 
in the technological field represented a second challenge for the teaching staff as they developed technological abilities, in general terms, for the pedagogical mediation; process which was not frequently done previous to the pandemic. (3) The selection of effective technological tools that were free-access and user-friendly for fostering meaningful learning. This essay concludes with reflective thoughts that reassure teaching practices and the challenges faced during the pandemic as well as opportunities for improvement in relationship with the adaptation, changes, and modernization processes throughout an uncertain and continuously-changing period of time.

Key Words: learning environment, teaching competences, technology, educational opportunities, digital gap, COVID-19

Resumo: Durante a pandemia gerada pelo vírus responsável pela COVID-19, o sistema educativo fez uma mudança completa do atendimento tradicional para o atendimento remoto; isto foi feito através da utilização de recursos tecnológicos e várias plataformas, o que permitiu a continuidade do processo educativo. Este evento mostrou que os modelos pedagógicos que estavam sendo implementados não respondiam às necessidades educativas de progresso e transformação digital geradas durante a crise da saúde. Portanto, este ensaio tem como principal objetivo refletir sobre os desafios e oportunidades de melhoria que os professores enfrentaram devido à pandemia. É desenvolvido sob uma abordagem interpretativa na qual são discutidos três desafios educacionais: (1) a mudança de contexto, ou seja, a migração das salas de aula para plataformas digitais e o seu impacto na comunicação entre professores e alunos; (2) a aprendizagem acelerada no campo tecnológico representou um segundo desafio para os professores tendo em conta a falta, em termos gerais, de capacidades tecnológicas para a mediação pedagógica, isto antes da pandemia; e (3) a escolha de ferramentas tecnológicas eficazes que fossem de livre acesso e fáceis de utilizar a fim de reforçar uma aprendizagem significativa. Este ensaio conclui com reflexões que reafirmam o trabalho académico e os desafios durante a pandemia, bem como as oportunidades de melhoria em relação à adaptação, mudança, e modernização num ambiente turbulento e incerto.

Palavras-chave: ambiente educativo, competências dos professores, tecnologia, oportunidades educativas, brecha digital, COVID-19

\section{INTRODUCCIÓN}

Durante el año 2020, Costa Rica, al igual que el resto del mundo, fue impactada por la pandemia causada por el virus responsable del COVID-19; esta situación, sin duda alguna, generó la necesidad de adoptar nuevas prácticas pedagógicas, lo cual repercutió en el campo docente de forma inesperada y repentina. El sistema educativo presencial no se encontraba completamente preparado y, en general, no respondía a las necesidades de progreso y transformación de la era digital. El ambiente turbulento y de emergencia suscitado a partir de la crisis sanitaria implicó repensar y tomar decisiones aceleradas acerca de alternativas educativas efectivas ante esta situación. Este proceso conllevó a una serie de cambios y desafíos relacionados con la mediación pedagógica que incidieron en los diferentes campos formativos desde el nivel preescolar hasta el universitario; es en este último ámbito en el que se enfoca el presente ensayo, el cual apunta hacia tres desafíos educativos específicos que el profesorado enfrentó durante los últimos meses.

Como punto de partida, y desde la experiencia universitaria, específicamente en la Universidad Nacional (UNA) y en la Universidad Estatal a Distancia (UNED), cabe resaltar que se abrieron espacios para el aprendizaje e intercambio de ideas a través de múltiples capacitaciones, cursos cortos, espacios colaborativos y seminarios en línea, cuyo propósito primordial fue enseñar y empoderar al cuerpo docente a utilizar plataformas y recursos educativos digitales que permitieran establecer un canal de comunicación efectivo para la mediación pedagógica. Desde esta perspectiva, se entiende que las herramientas tecnológicas se convirtieron en los aliados principales de comunicación, permitiendo así la generación de un nuevo espacio pedagógico, antes denominado aula o salón de clase, del cual deriva el primer desafío, llamado en este ensayo, cambio contextual.

Asimismo, un segundo reto importante al que se enfrentó el profesorado fue aprender de forma acelerada y eficiente a utilizar diferentes recursos tecnológicos, de modo tal que sus capacidades en esta área fueran factores que facilitaran y favorecieran su quehacer educativo y que permitieran una mediación pedagógica óptima; en este ensayo, este desafío ha sido nombrado como competencias tecnológicas. 
Finalmente, el personal académico afrontó un tercer reto educativo, la búsqueda de herramientas digitales de acceso libre y de fácil utilización que permitieran al estudiantado aprender, trabajar y presentar sus asignaciones de forma creativa, dinámica y efectiva.

En atención a lo anterior, este ensayo discute algunos de los desafíos generados en términos de la mediación pedagógica durante la pandemia generada por el virus responsable del COVID-19; esto con el objetivo de reflexionar acerca de los retos y oportunidades de mejora que el profesorado enfrentó recientemente en cuanto al cambio contextual, al aprendizaje y desarrollo de competencias tecnológicas, y a la escogencia de herramientas digitales funcionales.

\section{DESARROLLO}

\section{Cambio contextual}

Durante la pandemia, el cambio contextual fue, en definitiva, uno de los desafíos más grandes que enfrentó el sistema educativo costarricense. De acuerdo con la UNESCO-IESALC (2020), para abril, se estimaba que el cierre de centros educativos impactaría a más del 91.3\% de la población estudiantil mundial (párr. 2). En Costa Rica, el cierre preventivo de diferentes instituciones y la suspensión de actividades académicas en las universidades públicas, como es el caso de la Universidad Nacional (UNA) y de la Universidad Estatal a Distancia (UNED), entre otras, tomó lugar a inicios del mes de marzo de 2020.

Estas directrices fueron establecidas mediante varios acuerdos y un plan de contingencia institucional; en el caso de la UNA, se establecieron medidas tales como: migración de la presencialidad a la presencialidad remota, activación de protocolos en términos de capacitaciones para el profesorado durante el periodo comprendido entre el 20 de marzo y el 04 de abril de 2020 (inclusive), cambio de actividades en términos de mediación y evaluación de los aprendizajes para la modificación respectiva y migración a la presencialidad remota, disposición de plataformas de videoconferencias para las sesiones sincrónicas, apertura de repositorios o bases de datos académicas fundamentadas en material relacionado con la medicación pedagógica por medio de plataformas y el acceso a la tecnología, recolección de datos en cuanto a la conectividad estudiantil y recursos tecnológicos disponibles, activación de protocolos para brindar tarjetas SIM y tablets al estudiantado con limitaciones de conectividad, entre otros.

Por otra parte, en la UNED, se acordó solicitar al Programa de Teletrabajo preparar un plan de contingencia para incorporar temporalmente más personal, suspender todos los eventos masivos (como graduaciones, conferencias, y actividades estudiantiles), recomendar a las unidades académicas aprovechar las diversas facilidades tecnológicas disponibles, reducir las giras a los diferentes puntos del país, suspender las autorizaciones de viajes al exterior hasta que se normalizara la situación, así como realizar recordatorios a la comunidad universitaria acerca de las plataformas de video-comunicación con las que se contaban y la distribución respectiva de licencias y las orientaciones para su uso. Es así que estos acuerdos transformaron la manera en cómo el personal docente y el estudiantado se interrelacionaban en la UNA y la UNED. En esta línea, Didriksson et al. (2020) afirman que en el país:

Cada universidad debió organizarse según sus condiciones y recursos, de manera que no hubo una respuesta coordinada entre las 5 universidades estatales de las cuales solamente la Universidad Nacional Estatal a Distancia (UNED) se dedica fundamentalmente a la enseñanza en línea. Si bien esta modalidad se ha implementado en las otras universidades, no toda la población académica tenía instrucción, habilidades, o posibilidades para desarrollarla. Sin embargo, de un día para otro, sin haber conocido siquiera al estudiantado matriculado en sus cursos, ni tener los contactos, las y los docentes debieron trasladar todos sus cursos a plataformas virtuales. (p. 30) 
De la misma forma, García-Peñalvo, Corell, Abella-García, y Grande (2020) explican que el hecho de "suspender las actividades docentes presenciales en las universidades ocasionó que se transfirieran de modo inmediato todas ellas a un formato online" (p. 2), lo que ocasionó consecuencias que repercutieron en la mediación pedagógica. Este cambio contextual, es definido por Hodges, Moore, Lockee, Trust, y Bond (2020) como "Emergency Remote Teaching (ERT)" (párr. 6) o su traducción como enseñanza remota de emergencia, lo cual indicó ser una solución temporal ante la crisis sanitaria. Asimismo, Tijo (2020) recalca que "los espacios académicos que se han generado deben diferenciarse de lo que conocemos como cursos en línea y es más propio referirse a ellos como espacios académicos de Enseñanza Remota de Emergencia (ERE)" (p. 1). En otras palabras, gracias a los avances tecnológicos existentes tales como entornos virtuales, plataformas de videoconferencias, correos electrónicos, entre otras aplicaciones, fue posible, que tanto la UNA como la UNED pudieran restablecer la comunicación y mediar pedagógicamente el espacio de enseñanza-aprendizaje a pesar de la emergencia. Es decir, que tanto el personal docente como el estudiantado percibieran cercanía para continuar trabajando y aprendiendo, aún encontrándose en distintos espacios físicos.

Para algunos docentes y algunas docentes esto representó también un reto, ya que no contaban con el conocimiento previo requerido para la utilización de ciertas herramientas o su población estudiantil no disponía de los recursos tecnológicos necesarios para una comunicación fluida. En este sentido, la CEPAL y UNESCO (2020) afirma que:

Es preciso entender estas brechas desde una perspectiva multidimensional, porque no se trata solo de una diferencia de acceso a equipamiento, sino también del conjunto de habilidades que se requieren para poder aprovechar esta oportunidad, que son desiguales entre estudiantes, docentes y familiares a cargo del cuidado y la mediación de este proceso de aprendizaje que hoy se realiza en el hogar. Por lo mismo, es central que las políticas de promoción de un acceso más igualitario a la tecnología comiencen por reconocer estas diferentes dimensiones que estructuran las desigualdades sociales en la región y trabajar intencionalmente para revertirlas. (p.7)

En este escenario, cabe recalcar que la comunicación y la interacción efectiva durante la mediación pedagógica fueron de suma importancia para este nuevo paradigma. En palabras de Pérez (2009), la comunicación y la interacción en los contextos virtuales "propician el desarrollo de relaciones interpersonales que favorecen el aprendizaje y la cohesión del grupo, a través del establecimiento de objetivos comunes y redes de aprendizaje" (párr. 20). La misma promueve relaciones socio-afectivas que a su vez permiten la construcción de conocimiento cooperativo y reduce el sentimiento de soledad en el estudiantado (Pérez, 2009). Considerando esto, el cambio contextual a estas nuevas alternativas tecnológicas, más que un desafío, pudo ayudar a fomentar una interrelación social eficaz entre sus actores, generando así autonomía en el proceso de aprendizaje y espacios para el cumplimiento de objetivos colectivos.

Por otro lado, cabe señalar que el personal académico, en general, no contaba con entrenamiento previo relacionado con la utilización de recursos digitales para impartir lecciones totalmente de forma sincrónica y/o asincrónica; esto derivó en una serie de espacios de aprendizaje para comprender cómo la mediación pedagógica podía tomar lugar fuera del contexto educativo más común, el aula. Para esto, fue preciso que profesorado trabajara no solamente en el diseño y planeamiento de su lección regular, sino que también, estudiara cómo utilizar los recursos disponibles en plataformas digitales y sus características, y así hacer la incorporación respectiva en su plan de trabajo.

Asimismo, este proceso ocasionó un fenómeno realmente interesante en el contexto educativo de la UNA y de la UNED, esto, particularmente, en relación con la selección específica de materiales, actividades o estrategias para la mediación pedagógica, ya que, al impartir lecciones mediante una plataforma de videoconferencias, fue imperativo realizar dinámicas de trabajo que resultaran útiles y, principalmente, 
significativas para el proceso de aprendizaje del estudiantado. En este sentido, el profesorado debió embarcarse en la misión de explorar y diseñar materiales y buscar actividades o estrategias de mediación que permitieran mantener la atención de sus discentes y que, al mismo tiempo, promovieran la participación activa y la construcción del conocimiento de forma individual y grupal.

Es preciso resaltar que este nuevo paradigma fue bastante significativo, ya que el trasladarse de un espacio comúnmente establecido en "cuatro paredes" a nuevas áreas, las cuales variaron de hogar a hogar, fue todo un reto, debido a factores sociales, económicos, emocionales, entre otros, que entraron a regir en esta nueva dinámica de trabajo y estudio. Por ende, el cambio de contexto del aula tradicional a la mediación pedagógica digital o remota, más que un desafío representó oportunidades importantes de mejora para el sistema educativo nacional en cuanto a la comunicación, interacción, y participación entre el personal docente y la población estudiantil, así como en la capacitación docente y la escogencia de materiales, actividades, y estrategias significativas.

\section{Competencias tecnológicas}

La carencia de competencias tecnológicas fue otro desafío relevante que encaró el cuerpo docente ante la emergencia pandémica; en términos generales, esto provocó que el proceso de aprendizaje del personal académico en cuanto a la utilización eficaz de recursos digitales se diera de manera repentina y acelerada. Como bien afirma García-Peñalvo (2020), esta carencia se define como la brecha competencial en la educación digital, la cual fue más notoria en los últimos meses. En la misma línea, Prendes y Gutiérrez (2013) señalan que pese a que las universidades españolas toman acciones para apoyar al profesorado en la implementación de tecnología, el 70\% del personal docente admitió nunca o rara vez utilizar estos servicios de apoyo. Esta realidad no estuvo muy lejos de la costarricense y evidenció aún más el por qué de la carencia de capacidades tecnológicas impidiendo que el personal académico y estudiantado tomaran ventaja de los beneficios tecnológicos para una transformación digital.

Tal y como lo describen García-Peñalvo, Corell, Abella-García, y Grande (2020), esta situación de emergencia se dio de forma rápida y, para cierta parte del personal docente, sin el tiempo necesario para rediseñar cursos o programas presenciales y "sin poder planificar ni asegurar que todos los actores contaban con los medios tecnológicos mínimos requeridos, las competencias digitales necesarias y unas actitudes proclives al cambio" (p. 2). Consecuentemente, el proceso de aprendizaje y el desarrollo de competencias tecnológicas docentes, en particular, se dieron de manera abrupta, en donde la educación tradicional, usualmente criticada, podría finalmente abrir los espacios de cambio e innovación anteriormente tan demandados.

Según Sandí y Sanz (2018), las competencias tecnológicas también conocidas como competencias TIC o digitales, se pueden definir como el "conjunto de conocimientos y habilidades que tiene el personal docente y que le permiten el dominio adecuado de diversos recursos tecnológicos necesarios para su práctica docente" (p. 100). Sin embargo, durante el cambio contextual generado a partir de la pandemia, el profesorado se vio forzado no solamente a saber o conocer herramientas tecnológicas, sino también a utilizarlas apropiadamente durante la mediación pedagógica.

Ejemplo de esto son Estados Unidos y el Reino Unido, en donde se ha trabajado en el desarrollo de iniciativas sobre competencias tecnológicas presentes en el informe de la UNESCO (2004), el cual describe que el personal docente tecnológicamente competente debería ser capaz de implementar las siguientes competencias (citado en Prendes y Gutiérrez, 2013, p. 201):

a) Seleccionar y utilizar de forma apropiada una variedad de equipos y recursos tecnológicos con el objetivo de promover el aprendizaje. 
b) Ser usuarios competentes de una variedad de software y de herramientas tecnológicas y adaptarlas a la materia que enseñan y a la edad de sus alumnos.

c) Examinar de forma crítica la relevancia del software y de las herramientas tecnológicas para las materias que enseñan y juzgar su valor potencial en su aplicación en la clase.

d) Hacer uso constructivo de la tecnología de la información en sus clases y, en particular, preparar y poner en práctica planes de trabajo que incorporen de forma apropiada el uso de la tecnología.

e) Evaluar las formas en que el uso de la tecnología produce cambios en la naturaleza de la enseñanza y el aprendizaje.

Considerando las capacidades tecnológicas mencionadas, el personal docente, a pesar de enfrentar dificultades, también tuvo oportunidades de mejora y reflexión relacionadas con la importancia de dichas competencias para una mediación pedagógica transformadora y significativa. Monasterio y Briceño (2020) recalcan que "esta situación no fue planificada, pero se debe asumir como un aprendizaje" (p. 145), por lo que en la incertidumbre y emergencia actual, "es relevante que docentes y estudiantes adquieran las competencias tanto para aprender como para enseñar bajo la modalidad de educación sustentada por tecnologías" (p. 145). Por ende, en este nuevo contexto educativo, y pese a que autores como García-Peñalvo (2020) afirman que existe una resistencia al cambio por parte del profesorado y una carencia estratégica a nivel institucional, cabe resaltar que se generó una apropiación de competencias tecnológicas y la búsqueda de estrategias cooperativas entre docentes y las personas estudiantes para así facilitar y optimizar el funcionamiento de la labor pedagógica.

\section{Herramientas tecnológicas}

En el contexto vigente, el uso de herramientas de acceso libre y de fácil utilización fue el tercer desafío identificado en este ensayo. Didriksson et al. (2020) explican que tanto el estudiantado como el profesorado no contaban con el equipo o con acceso o conexión estable a internet para realizar la transición de la presencialidad a la educación remota, marcando también una brecha socioeconómica entre sus actores. A causa de esta situación, por ejemplo, en el caso de la UNA, se realizó una compra de 3000 tarjetas Sim y de equipos para su comunidad estudiantil (Didriksson et al., 2020, p. 30). Por otra parte, la UNED aprobó una serie de medidas para brindar soporte económico a la población estudiantil becada y no becada (Ramírez, 2020). Sin embargo, Didriksson et al. (2020) señaló que "mientras tanto, las y los profesores han debido ingeniárselas de manera individual para resolver las necesidades que siguen apareciendo" (p. 31). Este tercer desafío implicó que el personal académico invirtiera en licencias propias para plataformas digitales mientras la situación se estabilizaba. Otra parte del cuerpo docente optó por buscar herramientas tecnológicas de acceso libre y de utilización sencilla considerando que su población estudiantil contaba únicamente con dispositivos móviles (en particular, el celular).

Ante la carencia de herramientas tecnológicas, la búsqueda de estos recursos se convirtió en una necesidad inmediata para que el estudiantado pudiera contar con la posibilidad de trabajar de forma efectiva en las actividades académicas correspondientes. Mientras las universidades se encontraban trabajando en capacitar a la población universitaria y en habilitar el acceso a correos institucionales y sus contraseñas respectivas, el personal académico, por su parte, estaba en la búsqueda de herramientas digitales gratuitas (freeware) para ejecutar sus clases. Sin embargo, esto también presentó ciertas dificultades, por ejemplo, en el caso específico de las sesiones en vivo o sincrónicas, ya que algunas de estas plataformas de videoconferencias (con licencias gratuitas) contaban con un tiempo y capacidad de usuarios limitado, otras aplicaciones no permitían la creación de salas de interacción (break-out-rooms) dentro de las mismas, afectando así la mediación pedagógica. 
Considerando estos aspectos claves, la UNESCO-IESALC (2020), recomienda que las universidades faciliten el aprendizaje de la población estudiantil a través de sus plataformas y campus virtuales y, en caso de no poseerlas, sugiere brindar aplicaciones o plataformas educativas abiertas, tomando en cuenta que su estudiantado sólo pueda acceder por medio de dispositivos móviles a sus clases (párr. 8). En este sentido, la UNESCO (2020) afirma que las clases tienen que modificarse y que el tiempo de instrucción debería ser menor, ya que esto repercute en el éxito del aprendizaje; esto porque tanto para el cuerpo docente como para el estudiantado, no es factible tener lecciones en vivo o sincrónicas de tres a cuatro horas considerando que las personas estudiantes no siempre se encuentran en un contexto o espacio físico adecuado para aprender (párr. 6). Como resultado, esto conllevó a reflexionar que aparte de las actividades en vivo o sincrónicas sería más razonable considerar las actividades asincrónicas como parte importante en la mediación pedagógica así como contemplar la flexibilidad en la entrega de asignaciones y procesos de evaluación, no solo por el profesorado, sino también en las políticas y lineamientos institucionales (Hodges et al., 2020). Ciertamente, el uso de herramientas de acceso libre y fácil utilización fue uno de los desafíos presentes en la práctica docente durante la crisis sanitaria; no obstante, es lógico pensar que esta situación generó ideas de cambio que repercutirán de manera óptima en el futuro del campo educativo universitario.

\section{SÍNTESIS Y REFLEXIONES FINALES}

Si bien es cierto la pandemia generada por el virus responsable del COVID-19 impactó el sistema educativo costarricense, en el caso de este ensayo, a nivel universitario en la UNA y la UNED, se debe recalcar que las circunstancias y el hecho de no poder trasladarse al campus o centro de estudio generaron cambios positivos, ya que, durante la pandemia, se llevó el espacio pedagógico a casa, de forma tal, que el estudiantado continuara con su proceso de aprendizaje desde otro contexto. La imposibilidad de traslado repercutió en el cómo se mediaron las lecciones y fue de esta manera que el profesorado tuvo la oportunidad de aprender a utilizar diversas plataformas digitales y recursos educativos tecnológicos para afrontar el cambio contextual de ese momento.

Es relevante señalar que a pesar de que este cambio contextual fue súbito e imprevisto, el personal docente asumió un rol de aprendizaje a nivel tecnológico de forma rápida y efectiva, ya que tuvo que adaptar sus lecciones de manera adecuada y óptima procurando la utilización de los medios y recursos tecnológicos más apropiados para su contexto estudiantil particular; esto, indudablemente, le permitió ir más allá y conocer a sus discentes en términos del equipo y conectividad con el que contaban, así como diagnosticar aquellos aspectos favorables y poco favorecedores con los que había que lidiar desde un espacio como el hogar. En este sentido, fue imperante desarrollar una mediación pedagógica que, en la mayor medida posible, impactara significativamente el proceso de aprendizaje del estudiantado pero tomando como base su entorno particular.

A partir de la pandemia, otra oportunidad que surgió fue la modificación de la mediación pedagógica hacia un modelo más moderno, en el sentido del uso de herramientas tecnológicas que permitieran generar contenido digital amigable, atractivo, dinámico, y sencillo con el fin de transformar el contexto educativo hacia un ideal más innovador. Desde esta perspectiva, el cuerpo docente se enfrentó a un desafío que tiempo después se convertiría en la oportunidad y posibilidad de explorar y aprender a usar herramientas para generar contenido de clase y hacer un uso conveniente de recursos auditivos y visuales, tales como blogs, portafolios digitales, vídeos, wikis, entre otros. Este proceso permitió a las personas educadoras examinar plataformas y recursos digitales más allá de las frecuentemente utilizadas como procesadores de texto, presentaciones, y brochures o panfletos, comúnmente usadas a través de una licencia de Office. 
Asimismo, la búsqueda de algún espacio digital o repositorio para colocar información o asignaciones, le permitió al profesorado y le abrió la oportunidad de aprender o utilizar ese conocimiento para realizar sesiones en vivo o sincrónicas en plataformas de videoconferencias. Por ejemplo, en el caso específico de la UNA, se utilizaron las plataformas Microsoft Teams ${ }^{\oplus}$ y Zoom ${ }^{\circledast}$ (bajo licencias) y Google Meet ${ }^{\oplus}$, Jitsi ${ }^{\oplus}$, y Skype ${ }^{\oplus}$, como alternativas de acceso libre, así como espacios para almacenar información en la nube a través de medios como Drive, Dropbox ${ }^{\oplus}$, Google Classroom ${ }^{\oplus}$, Microsoft OneDrive ${ }^{\oplus}$, etc. Por otra parte, en el contexto educativo de la UNED, el personal docente contó con las plataformas BigBlueButton ${ }^{\oplus}$, Microsoft Teams ${ }^{\circledast}$ y WebEx ${ }^{\circledast}$ (mediante el uso de licencias). Así también, nació la ocasión de generar espacios de trabajo cooperativos en los cuales se organizaron equipos estudiantiles vinculados a un mismo recurso digital; por ejemplo, actividades o estrategias de mediación alojadas en Moodle, Google ${ }^{\circledR}$ o en espacios como Office $365^{\circledR}$, en los cuales la participación grupal fue el elemento clave.

Finalmente, la necesidad de utilizar herramientas tecnológicas y la carencia, en general, de licencias para el uso de las mismas, conllevó a la búsqueda de recursos digitales de acceso libre, de modo tal que las personas discentes pudieran contar con la posibilidad de trabajar en actividades o estrategias de mediación y en asignaciones de manera creativa, dinámica y efectiva. Este aspecto, en particular, apunta hacia la necesidad de dotar a la población con oportunidades que no generaran un costo adicional y que al mismo tiempo, fueran adecuadas y eficientes. Fue menester seleccionar también aquellos recursos que brindaran al usuario un diseño y características amigables; de modo tal, que el tiempo invertido estuviera altamente enfocado en el objetivo de la asignación o trabajo de clase, y no en dificultades relacionadas con el uso de una herramienta tecnológica específica.

En síntesis, el presente ensayo plantea tres desafíos enfrentados por el personal docente durante la pandemia generada por el virus responsable del COVID-19, los cuales podrían re-pensarse y ser concebidos como oportunidades de mejora para cambiar, modernizar, y optimizar el sistema educativo costarricense como respuesta ante la crisis sanitaria nacional. En este sentido, se propone al lector la posibilidad de profundizar en otras líneas investigativas relacionadas con esta temática, tales como: el impacto educativo generado a partir de la implementación de la presencialidad remota, la generación de políticas educativas relacionadas con el acceso a herramientas tecnológicas y protocolos de conectividad, la relevancia de generar protocolos educativos para afrontar situaciones de pandemia o de emergencia nacional que requieran un cambio contextual repentino, la preparación del personal académico en torno al desarrollo de competencias tecnológicas, entre otros. 
CEPAL y UNESCO. (2020). La educación en tiempos de la pandemia de COVID-19 (COVID-19 Informe CEPALUNESCO). Recuperado de https://unesdoc.unesco.org/ark:/48223/pf0000374075.locale=es

Didriksson, A., Álvarez, F., Caamaño, C., Caregnato, C., Sfredo, B., Del Valle, D. y Perrota, D. (2020). Educación superior y pandemia: ¿Innovamos, dilatamos el riesgo o perecemos? - reflexiones desde América Latina. Repositorio del Instituto de Investigaciones Sociales, 1-41. Recuperado de https:// repositorio.iis.ucr.ac.cr/handle/123456789/598

García-Peñalvo, F. (2020, 12 de mayo). El sistema universitario ante la COVID-19: Corto, medio y largo plazo [Mensaje en un blog]. Recuperado de https://www.universidadsi.es/ sistema-universitario-covid-19/

García-Peñalvo, F., Corell, A., Abella-García, V.y Grande, M. (2020). La evaluación online en la educación superior en tiempos de la COVID-19. Revista eUSAL, 12-26. Recuperado de https://repositorio.grial. eu/bitstream/grial/2010/1/a12.pdf

Hodges, C., Moore, S., Lockee, B., Trust, T. y Bond, A. (2020, 27 de marzo). The difference between emergency remote teaching and online learning [Mensaje en un blog]. Recuperado de https:// er.educause.edu/articles/2020/3/the-difference-between-emergency-remote-teaching-andonline-learning

Monasterio, D. y Briseño, M. (abril, 2020). Educación mediada por las tecnologías: Un desafío ante la coyuntura del Covid-19. Observador del Conocimiento, 5(1). Recuperado de http://www.oncti.gob. ve/ojs/index.php/rev_ODC/article/view/31/23

Pérez, M. (octubre, 2009). La comunicación y la interacción en contextos virtuales de aprendizaje. Apertura, 1(1). Recuperado de http://www.udgvirtual.udg.mx/apertura/index.php/apertura/ article/view/15/18

Prendes, M., \& Gutiérrez, I. (agosto, 2013). Competencias tecnológicas del profesorado en las universidades españolas. Revista de Educación, (361). Recuperado de https://www.educacionyfp.gob.es/ $\mathrm{dctm} /$ revista-de-educacion/articulosre361/re36108.pdf?documentld=0901e72b8162f031

Ramírez, K. (2020, 26 de mayo). UNED aprueba medidas de apoyo económico para estudiantes afectados por el COVID-19. Acontecer. Recuperado de https://www.uned.ac.cr/acontecer/a-diario/ gestion-universitaria/4064-uned-aprueba-medidas-de-apoyo-economico-para-estudiantesafectados-por-el-covid-19

Sandí, J., \& Sanz, C. (2018). Revisión y análisis sobre competencias tecnológicas esperadas en el profesorado en Iberoamérica. Edutec Revista Electrónica de Tecnología Educativa, (66), 93-121. https:// doi.org/10.21556/edutec.2018.66.1225

Tijo, S. (2020). Enseñanza remota de emergencia en ingeniería civil: Lecciones aprendidas. Encuentro Internacional de Educación en Ingeniería, 1-10. Recuperado de https://www.acofipapers.org/index.php/eiei/article/view/781

UNESCO. (2020). 290 millones de estudiantes sin clases por el COVID-19: La UNESCO divulga las primeras cifras mundiales y se moviliza para dar respuesta a la crisis. Recuperado de https://es.unesco.org/ news/290-millones-estudiantes-clases-covid-19-unesco-divulga-primeras-cifras-mundiales-yse-moviliza

UNESCO-IESALC. (2020). El Coronavirus Covid-19 y la educación superior: Impacto y recomendaciones. Recuperado de https://www.iesalc.unesco.org/2020/04/02/el-coronavirus-covid-19-y-la-educacion-superior-impacto-y-recomendaciones/ 